\title{
Probabilidades de internaÇão psiquiátrica de PaCientes de UM AMBULATÓRIO DE SAÚDE MENTAL
}

\author{
Leonardo Naves dos Reis ${ }^{1}$ \\ Julio Cesar Ribeiro Simplicio ${ }^{2}$ \\ Edilaine Cristina da Silva Gherardi-Donato ${ }^{3}$ \\ Ana Carolina Guidorizzi Zanetti ${ }^{4}$
}

\begin{abstract}
Objetivou-se avaliar fatores de predição (diagnósticos e características sociodemográficas) referentes à internação psiquiátrica entre usuários ambulatoriais de saúde mental. O estudo foi realizado a partir de dados secundários, extraídos dos prontuários e analisados por regressão logística, para obtenção da equação de predição da probabilidade de internação psiquiátrica. Os diagnósticos que apresentaram significância estatística $(p<0,05)$ foram transtorno afetivo bipolar, esquizofrenia, distúrbios ansiosos e depressão, e os dois primeiros apresentaram associação de alta magnitude com a necessidade de internação. A idade foi inversamente proporcional à necessidade de internação. Os resultados encontrados podem incitar ações específicas substitutivas e de prevenção à internação psiquiátrica aos pacientes mais jovens com esquizofrenia e transtorno afetivo bipolar.
\end{abstract}

Descritores: Saúde Mental; Epidemiologia; Hospitalização, Psiquiatria.

\footnotetext{
${ }^{1}$ Doutorando, Escola de Enfermagem de Ribeirão Preto, Universidade de São Paulo, Centro Colaborador da OPAS/OMS para o Desenvolvimento da Pesquisa em Enfermagem, Ribeirão Preto, SP, Brasil. Investigador de Polícia, Delegacia Seccional de Polícia de Sertãozinho, Sertãozinho, SP, Brasil.

2 Especialista em Informática em Saúde, Enfermeiro, Prevent Senior Private Operadora de Saúde, Ribeirão Preto, SP, Brasil.

3 PhD, Professor Associado, Escola de Enfermagem de Ribeirão Preto, Universidade de São Paulo, Centro Colaborador da OPAS/OMS para o Desenvolvimento da Pesquisa em Enfermagem, Ribeirão Preto, SP, Brasil.

${ }^{4}$ PhD, Professor Doutor, Escola de Enfermagem de Ribeirão Preto, Universidade de São Paulo, Centro Colaborador da OPAS/OMS para o Desenvolvimento da Pesquisa em Enfermagem, Ribeirão Preto, SP, Brasil.
} 


\section{Introdução}

Breve análise de estudos epidemiológicos sobre transtornos mentais pode mostrar o quanto essas doenças afetam a população mundial ${ }^{(1)}$. Em 2001, 450 milhões de pessoas sofriam de transtornos mentais, resultantes da interação de fatores genéticos e ambientais. Os transtornos mentais integram o quadro de doenças crônicas não transmissíveis e se apresentam como problema de saúde global e ameaça à saúde e ao desenvolvimento humano. As cargas dessas doenças recaem especialmente sobre países de baixa e média renda ${ }^{(2)}$.

No Brasil, com o advento da Constituição de 1988 e da Lei $n^{\circ} 8.080 / 90$, a qual estipulou os princípios do Sistema Único de Saúde (SUS), vivenciou-se um processo de transformações no modelo de assistência em saúde, inclusive no que se refere ao campo da saúde mental, quando o processo de saúde/doença passou a ser visto como processo social, o que tem trazido a consequente proposição das práticas de desinstitucionalização $0^{(3)}$.

Levando-se em conta os crescentes esforços direcionados à desinstitucionalização dos pacientes acometidos de transtornos psiquiátricos, e outros fatores como o aumento da expectativa de vida da população, pode-se inferir que tem ocorrido aumento na demanda e na importância dos serviços de níveis primário e secundário em saúde mental. Tais mudanças observadas trazem consigo alguns desafios a esses serviços, para que possam se adequar ao novo contexto e atender, de forma eficaz, as necessidades de sua clientela $^{(4)}$.

Um dos principais desafios consiste em conhecer o público atendido, conhecer o perfil dos usuários, em busca de uma caracterização que permita direcionar melhor as ações do serviço ${ }^{(5)}$.

Dentre os princípios que norteiam o SUS, destaca-se o da equidade, que busca diminuir as desigualdades, ao tratar os diferentes de forma diferente e o da integralidade que, entre outros aspectos, visa garantir o fornecimento de um conjunto articulado e contínuo de ações e serviços preventivos, curativos e coletivos em todos os níveis de assistência ${ }^{(6)}$. O conhecimento epidemiológico constitui importante ferramenta na manutenção desses princípios, uma vez que pode fornecer dados capazes de apontar peculiaridades do público atendido, bem como explicitar suas reais necessidades de saúde.

A importância da epidemiologia nos serviços de saúde se deve, em grande parte, ao seu papel na produção de conhecimentos para a tomada de decisões no que se refere à formulação de políticas de saúde, à organização do sistema e às intervenções destinadas a dar solução a problemas específicos. Dessa forma, funciona como valioso instrumento norteador do planejamento e realização das ações em saúde. Razões pelas quais o Ministério da Saúde classifica os sistemas de informações, tais como o Sistema de Informação da Atenção Básica (SIAB) e o Sistema de Informações Ambulatoriais (SIA), como instrumentos de gestão, tamanha a importância atribuída ao conhecimento epidemiológico ${ }^{(7-8)}$.

O convívio familiar e social é extremamente importante para a reabilitação do portador de transtorno mental e, nesse contexto, a internação psiquiátrica constitui a ruptura dessas relações ao trazer implicações para o decurso de sua vida cotidiana $^{(9)}$. A Lei brasileira ${ }^{\circ} 10.216 / 2001$, em seu artigo $4^{\circ}$, estabelece que um indivíduo deve ser hospitalizado em unidade de internação psiquiátrica, somente quando os demais tipos de tratamento tenham sido ineficazes ${ }^{(10)}$. Normalmente, as hospitalizações se dão após episódios de crises que acontecem por diferentes fatores, como abandono do tratamento e outros. Tais crises trazem como consequência ao doente não apenas aquelas relacionadas à internação, mas diversas outras ligadas ao processo da doença ${ }^{(11)}$. Dessa forma, é extremamente importante que a equipe de saúde realize, constantemente, intervenções preventivas direcionadas aos fatores que teriam como consequência a hospitalização do paciente e, para tanto, é fundamental que sejam conhecidos os fatores predisponentes, bem como os grupos de maior risco para que essas ações possam ser corretamente direcionadas. O conhecimento epidemiológico se apresenta como o instrumento que poderá fornecer subsídios importantes ao trabalho da equipe.

A necessidade de se prestar atendimento integral e equânime aos usuários de serviços de saúde mental, a carência de conhecimentos sobre o perfil epidemiológico da população atendida e, principalmente, a possibilidade de aumentar as 
chances de intervir junto aos pacientes portadores de transtornos mentais, no sentido de se evitar crises ou quaisquer outras manifestações que possam culminar em internação psiquiátrica, foram tais fatores que impulsionaram a execução deste estudo.

Objetivou-se avaliar fatores de predição (diagnósticos e características sociodemográficas) referentes à internação psiquiátrica entre usuários ambulatoriais de saúde mental. O estudo foi realizado a partir de dados secundários, extraídos dos prontuários e analisados por regressão logística, para obtenção da equação de predição da probabilidade de internação psiquiátrica.

O estudo também buscou conhecer as características sociodemográficas e diagnósticos desses pacientes, bem como, por meio da análise de regressão logística, a relação dessas características com a necessidade de internação psiquiátrica para, então, avaliar possíveis fatores de predição para hospitalização dos pacientes em estudo.

\section{Métodos}

Projeto aprovado pelo Comitê de Ética em Pesquisa da Escola de Enfermagem de Ribeirão Preto da Universidade de São Paulo (Protocolo $\mathrm{n}^{\mathrm{0}} 1446 / 2011$ ), de acordo com as normas estabelecidas pela Resolução 196/96, do Conselho Nacional de Saúde.

Realizou-se estudo quantitativo descritivo e exploratório, de natureza epidemiológica, de prevalência e correlacional. A amostra foi composta por todos os 1.281 indivíduos em tratamento no Núcleo de Saúde Mental (NSM) do Centro de Saúde Escola da Faculdade de Medicina de Ribeirão Preto, no período da coleta de dados, meses de abril e maio de 2012. Trata-se de estudo retrospectivo, baseado em dados secundários extraídos dos prontuários dos pacientes classificados como ativos no cadastro do serviço. O NSM é um serviço de natureza secundária e disponibiliza atendimento ambulatorial em saúde mental.

Foram consideradas as variáveis sexo, idade (anos completos), diagnóstico psiquiátrico (conforme classificação da CID-10) e internação. Essa última dividida nas categorias "sim", caso o paciente já tenha necessitado de pelo menos uma internação psiquiátrica, e "não", caso nunca tenha sido hospitalizado em razão de transtorno mental.

Na primeira etapa da análise de dados, realizou-se a estatística descritiva das características demográficas e diagnósticos dos pacientes, que já foram internados em unidade psiquiátrica ao menos uma vez ao longo da vida, comparando-as com as características do perfil geral do NSM. O grupo de pacientes que já necessitaram de internação psiquiátrica será denominado, neste estudo, como "grupo internação".

$\mathrm{Na}$ segunda etapa, foi testada a associação entre a variável considerada dependente, "internação" e as variáveis independentes (sexo, idade e os diversos diagnósticos). Para tanto, foi utilizada a análise de regressão logística com múltiplas variáveis e processo de seleção stepwise backward ${ }^{(12)}$.

De início, realizaram-se as análises univariadas entre a variável de desfecho (internação) e as variáveis independentes. Em seguida, foram incluídas, no modelo múltiplo, as variáveis exploratórias que apresentaram valor de $\mathrm{p}<0,20$ na análise univariada e foram mantidas no modelo final aquelas com $\mathrm{p}<0,05$ no modelo múltiplo.

Após a análise de regressão e obtenção do modelo múltiplo final, com as variáveis que se ajustaram ( $p<0,05)$, utilizaram-se os coeficientes obtidos, relativos a cada uma das variáveis independentes do modelo e elaborou-se a função logística referente à amostra, a qual possui como variável resposta à probabilidade de um paciente do NSM necessitar de internação psiquiátrica ao menos uma vez ao longo da vida. A mesma equação pode ser aplicada a pacientes novos no serviço como instrumento de predição da probabilidade de internação ${ }^{(12)}$. O modelo final obtido é exibido abaixo.

\section{Função logística (NSM)}

$\operatorname{logit}\left(p_{[i]}\right)=\beta_{0}+\beta_{1}$ Idade $+\beta_{2}$ Esquizofrenia $+\beta_{3}$ TAB $+\beta_{4}$ Depressão $+\beta_{5}$ T. de Ansiedade

$p_{[i]}$ : probabilidade de um paciente do NSM necessitar de internação ao menos uma vez ao longo da vida

$\beta_{0}$ : parâmetro constante, estimado com base nos dados amostrais $=-1,097663$ 
$\beta_{1}$ : parâmetro constante multiplicador da idade do paciente $=-0,0124421$

$\beta_{2}$ : parâmetro constante multiplicado por 1 , caso o paciente possua diagnóstico de esquizofrenia ou 0 , caso não possua $=1,151083$

$\beta_{3}$ : parâmetro constante multiplicado por 1 , caso o paciente possua diagnóstico de Transtorno Afetivo Bipolar (TAB) ou 0 , caso não possua $=1,195894$

$\beta_{4}$ : parâmetro constante multiplicado por 1 , caso o paciente possua diagnóstico de depressão ou 0 , caso não possua $=-0,8565831$

$\beta_{5}$ : parâmetro constante multiplicado por 1 , caso o paciente possua diagnóstico de transtorno de ansiedade ou 0 , caso não possua $=-1,437605$.

Uma vez elaborada a equação, obtiveram-se as retas relativas a cada transtorno, em função da idade e probabilidade de internação ${ }^{(12)}$.

Sintomas denominados somáticos apontam um processo ligado a uma cadeia causal, através da qual é possível inferir a existência de uma doença, no entanto, quando se trata de um sintoma psiquiátrico (não somático) os elementos a serem analisados, para que se possa chegar a um diagnóstico, são por excelência a experiência vivida pelo paciente, e essa é mediada por aspectos exteriores, ambientais. Esses "sintomas" muitas vezes constituem vivências muito particulares de cada indivíduo, o que dificulta afirmar que se trata de algo realmente ligado a algum processo patológico ${ }^{(13)}$. Por isso, o diagnóstico psiquiátrico depende de muitos fatores, entre eles a forma de interpretação de cada profissional $^{(14)}$. No NSM, a maioria dos pacientes é assistida por três psiquiatras diferentes, e uma pequena minoria por médicos residentes. É comum, no NSM, que um paciente que era atendido por determinado psiquiatra, após algum tempo, passe a ser atendido por outro, por diversas razões relacionadas à administração de recursos humanos do serviço de saúde, portanto, no prontuário de um mesmo paciente é possível observar que havia recebido determinado diagnóstico no início do tratamento e esse mudou ao longo do tempo, seja em razão de interpretações diferentes entre os psiquiatras ou porque as experiências vividas pelo paciente possibilitaram encontrar o diagnóstico mais adequado. Em razão dessas particularidades, ligadas ao diagnóstico psiquiátrico, foi admitido um viés relacionado à variável "diagnóstico" em que foi aceito o diagnóstico "atual", constante no prontuário do paciente, independentemente do profissional responsável pelo seu tratamento.

\section{Resultados}

Dentre todos os usuários em tratamento no NSM, 234 (18,27\%) já foram hospitalizados em unidades psiquiátricas, ao menos uma vez ao longo da vida. A comparação entre as distribuições por gênero e internação é exibida na Figura 1.

No que se refere à faixa etária, verificou-se, para o grupo internação, média de idade de 46,61 anos, enquanto que a média geral do NSM foi de 49,69 anos. Outro achado, o qual demonstra que os pacientes do grupo internação são, em geral, mais jovens é a pirâmide etária desses pacientes, a qual é deslocada à esquerda em relação à pirâmide do perfil geral do NSM, no entanto, chama atenção o baixo percentual de indivíduos de 18 a 29 anos, 7,95\% contra $17,59 \%$ da amostra geral de usuários do NSM. A grande maioria (76,15\%) dos pacientes do grupo internação possui idade de 30 a 59 anos (Figura 2).

Somados, os três diagnósticos mais prevalentes no grupo internação representam mais de $72 \%$ do total. A distribuição do grupo internação, segundo diagnóstico principal, é exibida na Figura 3.

Ao se analisar as taxas ajustadas, de cada patologia (Tabela 1), observa-se que o episódio maníaco e transtorno esquizoafetivo foram os diagnósticos que apresentaram maior proporção de pacientes que já necessitaram de internação, no entanto, nesse caso, a precisão da análise pode ter sido comprometida pela baixa prevalência desses diagnósticos na amostra. As proporções referentes aos demais transtornos também são exibidas na Tabela 1.

Por meio da análise de regressão logísitica com múltiplas variáveis, observou-se que havia associação entre a variável dependente "internação" e a variável independente "idade" $(p=0,003)$. Verificou-se, ainda, associação entre a variável internação e os diagnósticos esquizofrenia $(p<0,001)$, TAB $(p<0,001)$, depressão $(p=0,007)$ e distúrbios ansiosos $(p<0,001)$. 


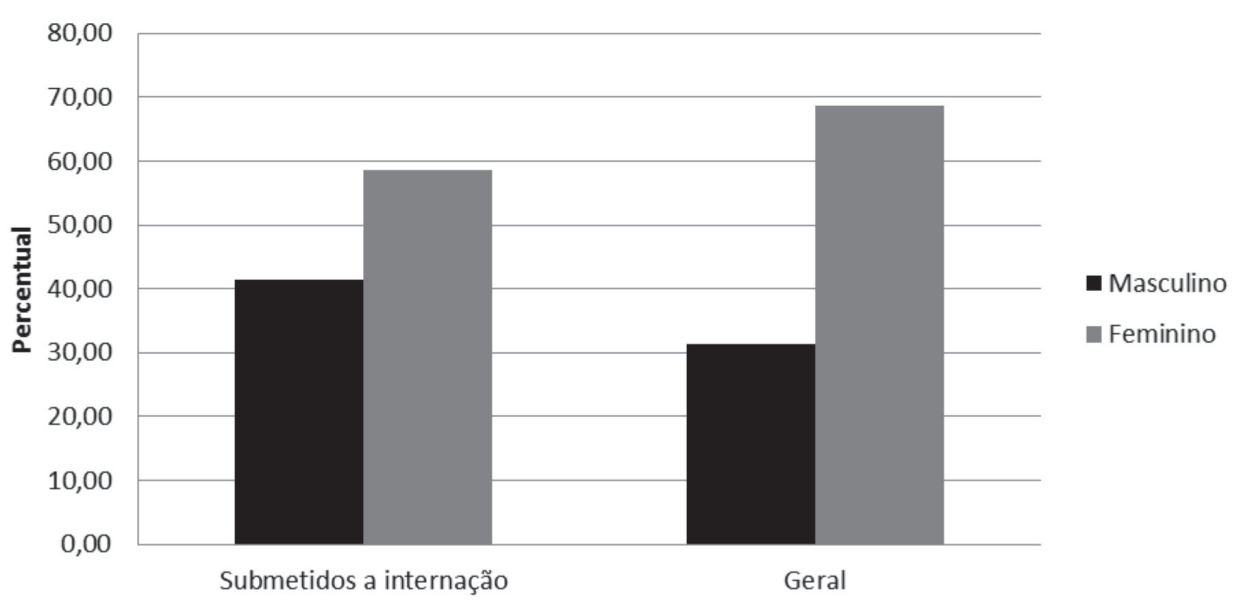

Figura 1 - Distribuições por gênero e internação do NSM. Ribeirão Preto, SP, Brasil, 2012

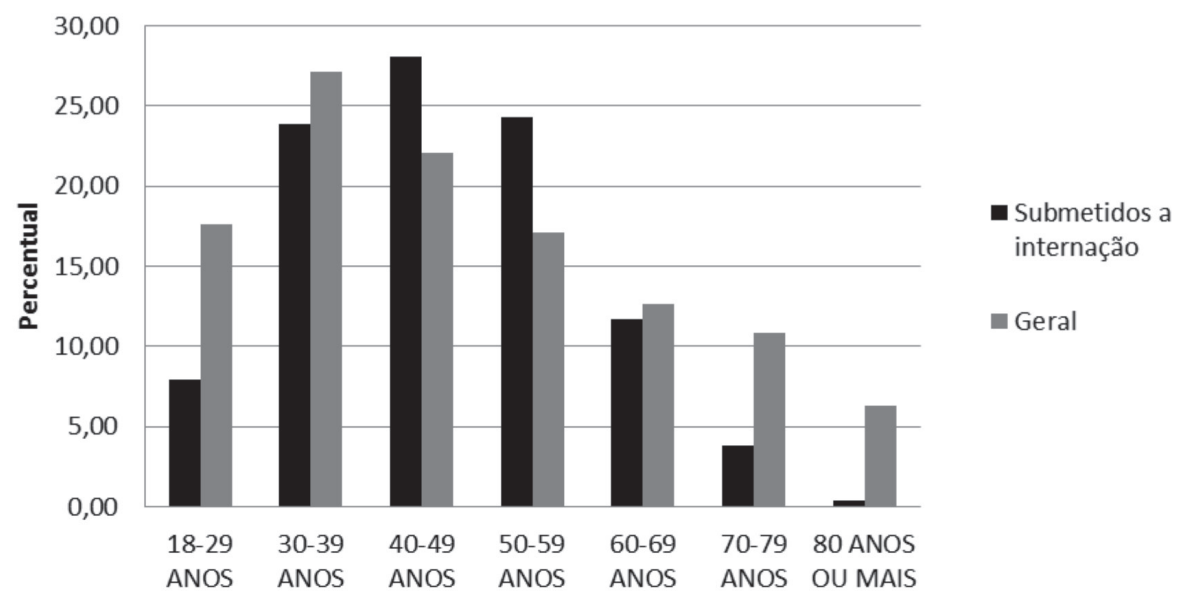

Figura 2 - Distribuição entre faixa etária e internações do NSM. Ribeirão Preto, SP, Brasil, 2012

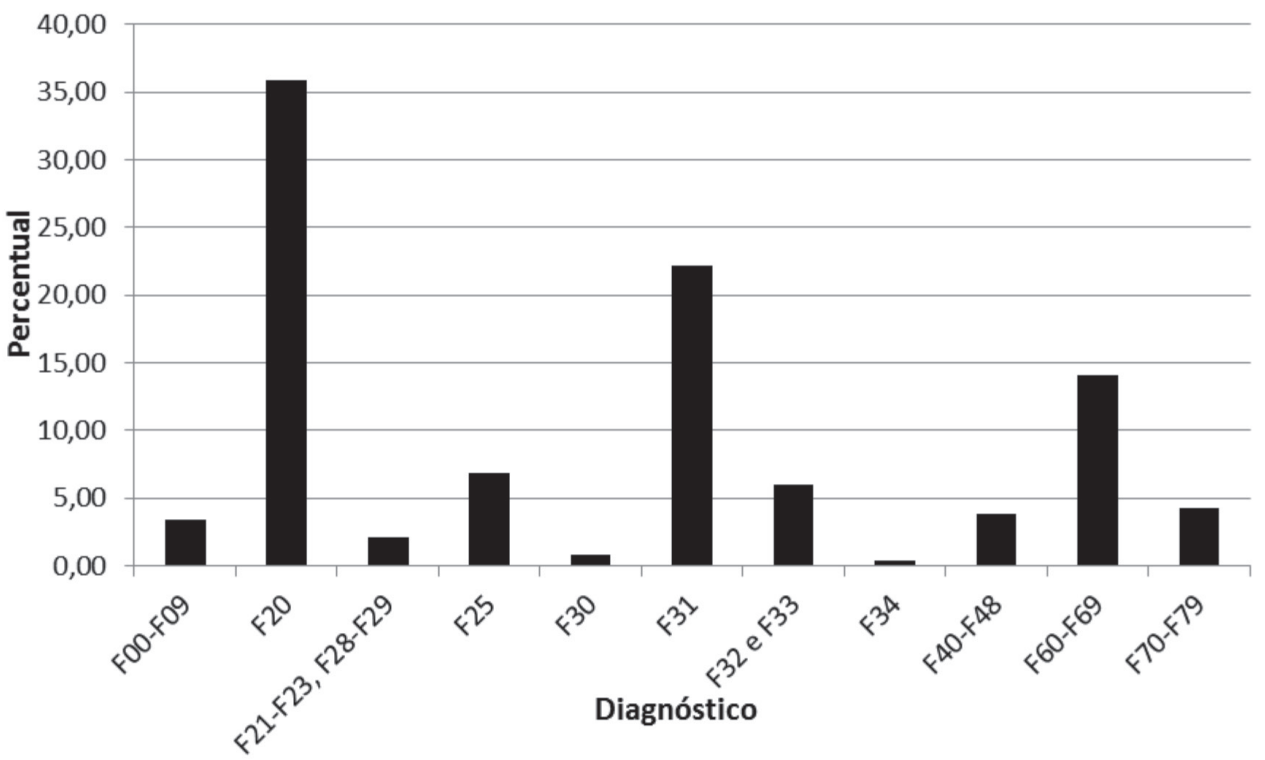

Figura 3 - Distribuição dos diagnósticos (DSM-IV) e internação psiquiátrica, ao menos uma vez ao longo da vida, segundo os pacientes do NSM. Ribeirão Preto, SP, Brasil, 2012 
Tabela 1 - Taxa de usuários do NSM que já foram submetidos a internação ao menos uma vez ao longo da vida, segundo diagnóstico (usuários submetidos a internação/100 pacientes). Ribeirão Preto, SP, Brasil, 2012

\begin{tabular}{lc}
\hline Diagnóstico principal & Submetidos a internação/100 pacientes \\
\hline F00-F09 & 22,22 \\
F10-F19 & 0,00 \\
F20 & 36,52 \\
F21-F23, F28-F29 & 10,87 \\
F25 & 41,03 \\
F30 & 66,67 \\
F31 & 37,96 \\
F32 e F33 & 4,88 \\
F34 & 5,26 \\
F40-F48 & 4,25 \\
F60-F69 & 16,18 \\
F70-F79 & 18,87 \\
G40 & 0,00 \\
Não informado & 0,00 \\
Total & 18,27 \\
\hline
\end{tabular}

Assim como em relação à variável "sexo", para transtornos de origem orgânica, transtornos relacionados ao uso de substâncias, transtornos equizotípico, delirantes e psicóticos, episódio maníaco, transtornos de personalidade e retardo mental, não foi possível observar associação $(\mathrm{p}>0,05)$.

Após a elaboração da função logística da população de pacientes do NSM, a qual continha os coeficientes relativos à idade e os transtornos que se adaptaram ao modelo de regressão, foi possível traçar as retas referentes à probabilidade de necessidade de internação em função do diagnóstico e idade (Figura 4).

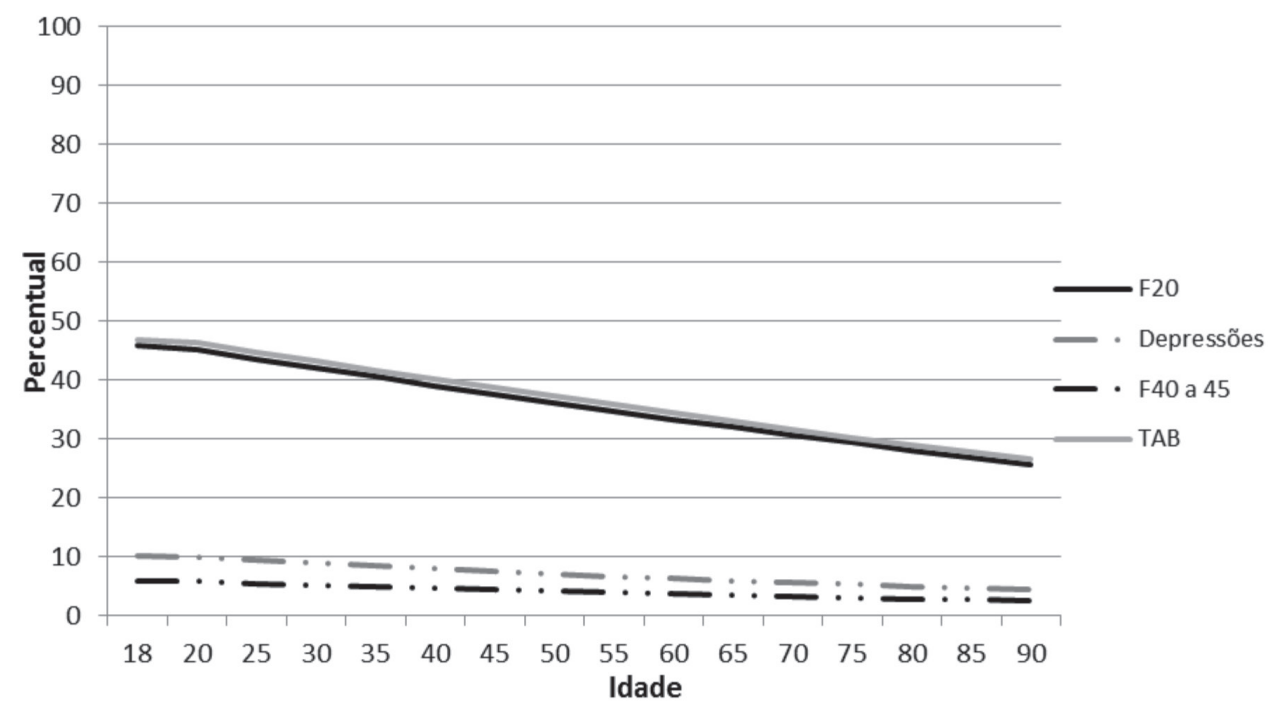

Figura 4 - Probabilidade (\%) de um usuário do NSM necessitar de internação psiquiátrica, ao menos uma vez ao longo da vida, segundo idade e transtorno mental. Ribeirão Preto, SP, Brasil, 2012

\section{Discussão}

Análises direcionadas às características sociodemográficas, realizadas em hospitais psiquiátricos, apontam que a maioria dos pacientes institucionalizados são do sexo masculino, e observou-se que os homens representam entre 53 e $61 \%$ do total ${ }^{(15-16)}$. Já entre os pacientes do NSM que já necessitaram de internação psiquiátrica, observa-se o oposto, visto que as mulheres compõem a maioria, aproximadamente $59 \%$. Outro estudo realizado no NSM, no ano 2007, já apontava que os indivíduos do sexo feminino eram a maioria entre os egressos de internação psiquiátrica, e representavam cerca de $62 \%$ do total $^{(11)}$.

No que diz respeito aos transtornos mentais, vários autores defendem a ideia do gênero como construção psicossocial que influenciará inevitavelmente a expressão da saúde mental. Em alguns transtornos, homens e mulheres podem apresentar diferenças relacionadas ao prognóstico, às taxas de suicídio, ao tempo de internação e ao número de reinternações psiquiátricas. Tais diferenças entre os gêneros sugerem a necessidade de abordagens diferentes por parte das equipes de saúde mental, em função do sexo. Sabe-se que, em vários países, o planejamento 
de intervenções é diferenciado para homens e mulheres ${ }^{(16-17)}$. E, nesse contexto, o conhecimento acerca do perfil dos usuários se torna extremamente relevante, como ferramenta de gestão e planejamento das intervenções específicas para indivíduos de ambos os sexos.

Estudo, no qual se verificou a relação das características sociodemográficas e dignósticos com o desfecho positivo ou negativo da internação psiquiátrica, aponta que os indivíduos acima de 60 anos possuem maior chance de apresentar desfecho desfavorável do que os mais jovens ${ }^{(18)}$. Praticamente, $30 \%$ dos pacientes do NSM encontram-se na faixa etária de 60 anos ou mais. Há, ainda, outro trabalho o qual demostra que quanto maior a idade do paciente maior a incidência de reinternações psiquiátricas ${ }^{(16)}$. Entre os usuários que já foram submetidos a internação, apenas $31,8 \%$ possuem idade abaixo de 40 anos.

Dados como os descritos no parágrafo anterior chamam atenção, no que se refere ao contexto específico do NSM, para a necessidade de ações no sentido de se evitar as reinternações, tendo em vista a média de idade dos pacientes do grupo "internação", relativamente próxima de 50 anos, e o grande número de pacientes com idade acima de 40 anos. Há de se atentar ainda que número significativo de usuários do serviço possuem idade acima de 60 anos e. como já descrito, estss pacientes possuem maior chance de desfecho negativo após as internações.

Chama atenção, na Tabela 1, as altas taxas de internação observadas para os pacientes com diagnóstico de esquizofrenia, TAB e, em especial, transtornos de personalidade, tendo em vista que, para esse último diagnóstico, não se observou associação estatisticamente significativa após a análise de regressão logística. No entanto, a análise simplesmente descritiva, exibida na referida Tabela, não permite falar em associação ou relação causal entre os diagnósticos e a internação, porém, poderia incitar pesquisas mais aprofundadas no NSM, com a finalidade de se investigar tal associação, salientando, ainda, que estudos recentes também demonstram que percentual importante dos pacientes acometidos por transtornos de personalidade acabam por necessitar de hospitalização psiquiátrica ${ }^{(11,19)}$. Importante ressaltar, também, que entre os transtornos psiquiátricos, esquizofrenia, TAB e transtornos de personalidade, estão aqueles que constituem maior fator de risco ao suicídio, assim como a depressão, conforme aponta estudo de coorte realizado na Suécia ${ }^{(20)}$. Tais considerações remetem também à necessidade de ações específicas, por parte da equipe de saúde, direcionadas a esse tipo de paciente, com a finalidade de prevenir as "crises" que poderiam culminar em hospitalização do paciente.

Estudos, realizados no Brasil, apontam como diagnóstico mais frequentemente verificado entre os pacientes institucionalizados o grupo das esquizofrenias e transtornos associados (percentuais entre 43 e $54 \%)^{(21-23)}$, percentual muito próximo ao verificado entre os pacientes do NSM que já foram hospitalizados $(44,87 \%)$, no entanto, estudos realizados na Itália e China, apontam a depressão como a causa mais frequente de internação ${ }^{(24-25)}$. Em específico, no caso do serviço em estudo, e talvez ainda no caso do Brasil, em razão dos resultados obtidos em outros estudos nacionais ${ }^{(21-23)}$, há de se atentar para ações preventivas no sentido de se evitar reinternações dos pacientes portadores de transtorno mental, em especial os esquizofrênicos, tendo em vista que são maioria entre aqueles que já necessitaram de internação e constituem o perfil de pacientes mais suscetíveis a reinternações ${ }^{(16)}$.

Por meio da observação da Figura 4, verifica-se que a variabilidade relacionada à probabilidade de internação dos pacientes do NSM é ampla, e que, por exemplo, um paciente com 18 anos de idade e diagnosticado com TAB apresenta probabilidadade de aproximadamente $47 \%$. Já um paciente com 65 anos de idade e que sofra de algum distúrbio ansioso apresenta pouco mais de $3 \%$ de chances de necessitar de internação. A mesma variabilidade é observada na Tabela 1, a qual demonstra que, entre aqueles com transtornos de ansiedade, de cada 100 apenas 4,25 já foram internados, enquanto que, em relação aos pacientes com TAB, essa taxa é quase nove vezes maior.Pôde-se notar que pacientes mais jovens e portadores de determinados transtornos demandam maior atenção no sentido de se prevenir as internações psiquiátricas.

Verificou-se, também, que os transtornos que apresentam maior probabilidade de internação são o TAB e a esquizofrenia, assim como se pode verificar em outros estudos que tratam sobre internação psiquiátrica ${ }^{(11,19-20)}$. Tal achado, certamente, está relacionado aos sintomas dessas patologias, os quais trazem grande prejuízo ao funcionamento do indivíduo, muitas vezes suficiente para gerar sua hospitalização ${ }^{(11)}$. 
Estudos como os mencionados no parágrafo anterior sugerem, em relação aos pacientes com diagnóstico de TAB ou esquizofrenia, cuidados no sentido de serem prevenidas as crises que poderiam culminar em hospitalização, o que, como consequência, acabaria ceifando-os do convívio familiar e social, tão importantes para a reabilitação desses indivíduos.

Importante salientar que os achados deste trabalho divergem de outros observados no âmbito internacional como, por exemplo, em um desses trabalhos, no qual os autores correlacionam utilizações dos serviços de saúde com condições de saúde, além dos resultados expostos, menciona-se sobre como é ampla a produção literária no sentido de afirmar que a depressão está associada ao aumento da utilização de serviços de nível terciário $^{(26)}$. Os resultados obtidos nesse estudo, no que se refere à depressão, muito provavelmente, estão relacionados a alguma particularidade do serviço de saúde investigado, o que poderia motivar outros estudos no mesmo local, com a finalidade de entender tais particularidades.

Ainda que a probabilidade de internação dos pacientes acometidos por depressão possa ser considerada baixa, quando comparada, por exemplo, ao TAB e à esquizofrenia (Figura 4), deve haver especial atenção da equipe de saúde a essa clientela, especialmente a clientela mais idosa. A depressão está entre as doenças crônicas que mais atingem essa população e depreciam sua qualidade de vida ${ }^{(27)}$, além disso, o transtorno depressivo constitui fator de risco significativo para o suicídio, principalmente em indivíduos que necessitaram de atenção em nível hospitalar ${ }^{(26)}$.

\section{Conclusão}

Os resultados do presente estudo podem servir para direcionar as ações preventivas e substitutivas às internações psiquiátricas, por parte do serviço de saúde, e poderiam, por exemplo, incitar cuidado mais próximo aos pacientes jovens com diagnóstico de esquizofrenia ou TAB, talvez um programa permanente de visitas domiciliares aos pacientes com perfil de maior risco para internações ou, ainda, o agendamento de retornos mais frequentes desses pacientes ao serviço.

Em um momento em que tem se falado tanto em reforma psiquiátrica, desinstitucionalização e reabilitação psicossocial, ações preventivas, no sentido de se evitar situações que acabariam por culminar em internações psiquiátricas, se tornam extremamente importantes. Tal entendimento, aliado ao conhecimento epidemiólogico, se torna ferramenta valiosa de gestão em saúde mental. As ferramentas de predição, no caso deste estudo especialmente relacionado às internações psiquiátricas, podem servir como instrumento norteador das ações da equipe no sentido de racionalizar a aplicação dos diversos tipos de recursos e em busca do atendimento equânime e integral, segundo os princípios do Sistema Único de Saúde.

\section{Limitações}

Por fim, são consideradas limitações do presente estudo a utilização de dados secundários de registros médicos, não apenas pelo fato de não se ter a coleta diretamente com o sujeito pesquisado, mas, também, em razão da não padronização dos critérios de diagnósticos, já que no serviço havia mais de um médico psiquiatra, e a interpretação dos sinais e sintomas que levam ao diagnóstico final pode variar entre os profissionais. Outra importante limitação está relacionada ao tipo de estudo (transversal), sendo que melhor seria se tivesse havido a possibilidade da realização de um estudo de coorte, porém, os recursos disponíveis inviabilizavam esse tipo de trabalho.

\section{Referências}

1. Serrano-Blanco A, Palao DJ, Luciano JV, Pinto-Meza A, Luján L, Fernández A, et al. Prevalence of mental disorders in primary care: results from the diagnosis and treatment of mental disorders in primary care study (DASMAP). Soc Psychiat Epidemiol. 2010;45:201-10.

2. Silva DS, Santana PR. Transtornos mentais e pobreza no Brasil: uma revisão sistemática. Tempus - Actas Saúde Coletiva. 2012;6(4):175-85.

3. Parente ACM, Menezes LC, Castelo Branco FMF, Sales JCS, Parente ACBV. Reform of Psychiatric care in Brazil: reality and perspective. REUFPI. 2013;2(2):66-73.

4. Feitosa CMA, Silva T, Silveira MFA, Santos Junior HPO. (Re)construção das práticas em saúde mental: Compreensão dos profissionais sobre o processo de desinstitucionalização. Psicol Teor Prat. 2012;14(1):40-54.

5. Tomasi E, Facchini LA, Piccini RX, Thumé E, Silveira DS, Siqueira FV, et al. Perfil 
sócio-demográfico e epidemiológico dos trabalhadores da atenção básica à saúde nas regiões Sul e Nordeste do Brasil. Cad Saúde Pública. 2008;24(1):193-201.

6. Ayres JRCM, Carvalho YM, Nasser MA, Saltão RM, Mendes VM. Caminhos da integralidade: adolescentes e jovens da Atenção Primária à Saúde. Revista Interface - Comunicação, Saúde e Educação. 2012; 16(40):67-81.

7. Domingos PAS, Ribeiro DG, Dinelli W, Staufackar CA, Campos JABD. Aspectos epidemiológicos da saúde bucal de crianças em um município brasileiro. Arq Odontol. 2010; 45(2):82-7.

8. Almeida GCM, Ferreira MAF. Saúde bucal no contexto do Programa Saúde da Família: práticas de prevenção orientadas ao indivíduo e ao coletivo. Cad Saúde Pública. 2008; 24(9):93-104.

9. Ramos DKB, Guimarães J, Bertha CE. Análise contextual de reinternações frequentes de portador de transtorno mental. Interface - Comun Saúde Educ. 2011;15(37):519-27.

10. Lei 10216, de 6 de abril de 2001 (BR). Dispõe sobre a proteção e os direitos das pessoas portadoras de transtornos mentais e redireciona o modelo assistencial em saúde mental. Diário Oficial da União. Poder Executivo. Brasília; 2001.

11. Cardoso L, Galera SAF. Quem são os egressos de internação psiquiátrica? Acta Paul Enferm. 2009;22(6):733-40.

12. Pagano M, Gauvreau K. Princípios de Bioestatística.São Paulo: Editora Cengage Learning; 2010.

13. Bloc L, Moreira V. Sintoma e fenômeno na psicologia fenomenológica de Arthur Tatossian. Rev Latinoam Psicopatol Fund. 2013;16(1):28-41.

14. Ferreira LO. Jurupari ou "visagens": reflexões sobre os descompassos interpretativos existentes entre os pontos de vista psiquiátrico e indígenas. Medicações. 2012;16(2):249-65.

15. Oliveira MSN, Pinto FJM, Aguiar JB, Sampaio RMM, Medeiros CRB. Perfil sociodemográfico e clínico de pacientes em internações psiquiátricas voluntárias e involuntárias. Rev Bras Promoç Saúde. 2011;24(4):361-6.

16. Castro AS, Furegato ARF, Santos JLF. Características sociodemográficas e clínicas em reinternações psiquiátricas. Rev. Latino-Am. Enfermagem. 2010;18(4):800-8.

17. Rabasquinho C, Pereira H. Gênero e saúde mental: Uma abordagem epidemiológica. Análise Psicol. 2007;3(25):439-54.
18. Sousa-Muñoz RLS, Tagushi LC, Martins GCCS, Andrade MR, Lima KMSR. Prevalência e fatores associados com ocorrência de delirium em adultos e idosos internados. Rev Bras Clin Med. 2012;10(4):285-90.

19. Furlan PM, Paschetta E, Solia F, Picci RL, Giaretto R, Carezana C, et al. O serviço psiquiátrico de diagnóstico e tratamento no hospital geral: usuários e novas urgências. J Nurs Health. 2011;1(1):300-10.

20. Tidemalm D, Langstrom N, Lichtenstein P, Runeson B. Risk of suicide after suicide attempt according to coexisting psychiatric disorder: Swedish cohort study with long term follow-up. BMJ. 2008;337:a2205.

21. Paixão C, Matias D, Alencar I, Nunes M, Sales P, Veiga PHA. Análise da prevalência dos transtornos psíquicos na região metropolitana do Recife. Ciênc Saúde Coletiva. 2009; 14(1):261-6. 22. Kilsztajn S, Lopes ES, Lima LZ, Rocha PAF, Carmo MSN. Leitos hospitalares e reforma psiquiátrica no Brasil. Cad Saúde Pública. 2008;24(10):2354-62.

23. Souza J, Souza N, Magna L. Tempo médio de hospitalização e categorias diagnósticas em hospital psiquiátrico. J Bras Psiquiatria. 2008;57(2):112-6.

24. Phillips MR, Zhang J, Shi Q, Song Z, Ding $Z$, Pang S, et al. Prevalence, treatment, and associated disability of mental disorders in four provinces in China during 2001-05: an epidemiological survey. Lancet. 2009;373(9680):2041-53.

25. Rocca $\mathrm{P}$, Mingrone $\mathrm{C}$, Mongini $\mathrm{T}$, Montemagni C, Pulvirenti L, Rocca G, et al. Outcome and length of stay in psychiatric hospitalization, the experience of the University Clinic of Turin. Soc Psychiatr Psychiatric Epidemiol. 2010;45(6):603-10.

26. Ballard ED, Cwik M, Storr CL, Goldstein M, Eaton WW, Wilcox HC. Recent medical service utilization and health conditions associated with a history of suicide attempts. Gen Hosp Psychiatry. 2014;36:437-41.

27. Garcia MAA, Rodrigues MG, Borega RS. O envelhecimento e a saúde. Rev Ciênc Méd. 2012;11(3):221-31. 